http://dx.doi.org/10.18778/2196-8403.2008.21

\title{
THEMATISCHER SCHWERPUNKT 2010: Aggressivität in Sprache und Literatur
}

Sprache hat immer auch eine emotionale Dimension. Convivium will sich im Schwerpunkt des Jahrgangs 2010 mit der aggressiven, der dunklen Seite von Sprache und Literatur auseinandersetzen, die erst in den letzten Jahren wieder verstärkt in den Fokus des wissenschaftlichen Interesses gerückt ist.

In der Sprachwissenschaft geht es dabei um Sprache als Instrument zur Ausübung von Gewalt.

Folgt man den Medienberichten, gehören Gewalttaten zu unserem Alltag undenkbar wären die Massenmedien ohne stereotype Meldungen über Gewalt z. B. von randalierenden Jugendlichen, von Einbrüchen und Überfällen. Sie vermitteln den politisch oft gewollten Eindruck eines permanenten Anstiegs der Fälle unmittelbar physischer Aggression. Tatsächlich aber ereignen sich in unserem Alltag primär Konfliktsituationen und Gewaltakte, die sich auf der sprachlichen Ebene abspielen - mit Sprache werden Menschen angegriffen, verletzt und gedemütigt; ihnen wird Schaden durch Worte zugefügt (physische Gewalt resultiert oft erst daraus). Gewalt in und durch Sprache schenkt man deshalb in der linguistischen Forschung immer mehr Aufmerksamkeit. Sie gilt als mehrdimensionales Untersuchungsobjekt. Entsprechend kann bei Beiträgen zu diesem Schwerpunktthema auf verschiedene Aspekte der sprachlichen Aggression eingegangen werden; zum einen kann das Inventar der Sprachmittel untersucht werden, mit deren Hilfe Gewalt ausgeübt wird; zum anderen bietet sich die Möglichkeit, mit (pragma- und/oder text-)linguistischen Methoden Akte der Sprachgewalt in unterschiedlichen kommunikativen Bereichen, in verschiedenen Medien und auch in unterschiedlichen Textsorten $\mathrm{zu}$ untersuchen.

Auch für die Literaturwissenschaft ist das Thema „Aggressivität“ mehrfach interessant: Nicht nur ist Aggression ein notwendiges Moment literarischer Darstellungen, Spannung in Drama oder Roman z. B. wäre kaum möglich ohne (offen oder verdeckt) aggressiv vertretene unterschiedliche Interessen handlungstreibender Figuren. Oft motiviert die Verarbeitung als traumatisierend erfahrener realer Gewalt überhaupt erst autobiographisches, lyrisch-expressives oder fiktionales Schreiben. Die motivliche Palette reicht von familiärem, schulischem, betrieblichem oder politischem Psychoterror über Freiheitsberaubung, Vergewaltigung und physischer Gewaltanwendung bis hin zu Krieg und Genozid. Aus literaturwissenschaftlicher Sicht brisanter noch wäre freilich die Frage, inwieweit Literatur Aggression nicht nur darstellen und behandeln, son- 
dern selbst aggressiv sein kann - sei es durch den Umgang von Erzählern oder Autoren mit ihren Figuren (etwa in Karikatur oder propagandistischer Negativzeichnung) oder impliziten Lesern (man denke nur an gezielte Tabubrüche und Schockeffekte aller Art), sei es durch die Art des Sprachgebrauchs oder der Textkomposition selbst. Aggression kann Thema von Literatur sein, Aggressivität eine Eigenschaft von Literatur.

Wer sich mit einem Aspekt dieses breiten Themenspektrums beschäftigen und dazu einen Beitrag für Convivium 2010 verfassen möchte, wird gebeten, der Redaktion bis zum 30. Juni 2009 einen vorläufigen Titel mitzuteilen und den Beitrag bis zum 30. November 2009 einzureichen. Die „Hinweise zur Einrichtung des druckfertigen Manuskripts“ können unter www.convivium.pl eingesehen werden. 